\title{
Viewpoint
}

\section{Benefits of a roundabout}

\author{
Gavin W. Morley \\ London Centre for Nanotechnology and Department of Physics and Astronomy, University College London, \\ 17-19 Gordon Street, London WC1H OAH, UK
}

Published January 19, 2010

The signal-to-noise ratio for room-temperature readout of individual qubits can be substantially improved by taking an indirect route through intermediate energy levels.

\section{Subject Areas: Quantum Information}

\author{
A Viewpoint on: \\ Universal enhancement of the optical readout fidelity of single electron spins at nitrogen-vacancy \\ centers in diamond \\ M. Steiner, P. Neumann, J. Beck, F. Jelezko, and J. Wrachtrup \\ Physical Review B 81, 0352052010 - Published January 19, 2010
}

Electronic and nuclear spins in insulating crystals interact weakly with their environments, making them candidate systems for applications in quantum information processing [1, 2]. Several features of the nitrogen vacancy $\left(\mathrm{NV}^{-}\right)$center in diamond (see Fig. 1) make it ideal for this purpose, as well as for sensitive magnetometry [3, 4]: The electron spin has been shown [5] to store quantum information for longer than any other solid-state system at room temperature. Additionally, this spin state can be partially initialized to a useful starting state simply by the application of light [6]. The same light excites spin-dependent fluorescence [7] bright enough to study single centers at room temperature [8]. Performing these experiments at low temperatures has even made it possible to readout the spin state of a single center [9]; to qualify as spin state readout, the signal averaging must be completed before the spin state information is destroyed.

For room-temperature single $\mathrm{NV}^{-}$demonstrations to date, the signal-to-noise ratio obtained before destroying a spin state is only $\sim 0.1$, preventing single spin state readout, and requiring signal averaging over many runs. The desire to go beyond this has led several groups to develop novel readout techniques. One recent technique uses nearby isotopes, ${ }^{13} \mathrm{C}$ nuclear spins, to store the electronic spin state before using the same electronic spin to repeatedly readout the state of the ${ }^{13} \mathrm{C}$ spins $[10$. This repeated readout technique works because the nuclear spins are disturbed less than the electron spins by the fluorescence measurement process. An improvement in the signal-to-noise by a factor of 2.2 was hence shown, but a different $\mathrm{NV}^{-}$center with a different distribution of nearby ${ }^{13} \mathrm{C}$ spins would require a modified technique and produce different signal-to-noise.

A paper published in the latest issue of Physical Review $B$ presents an alternative readout technique that

DOI: $10.1103 /$ Physics.3.6

URL: http://link.aps.org/doi/10.1103/Physics.3.6

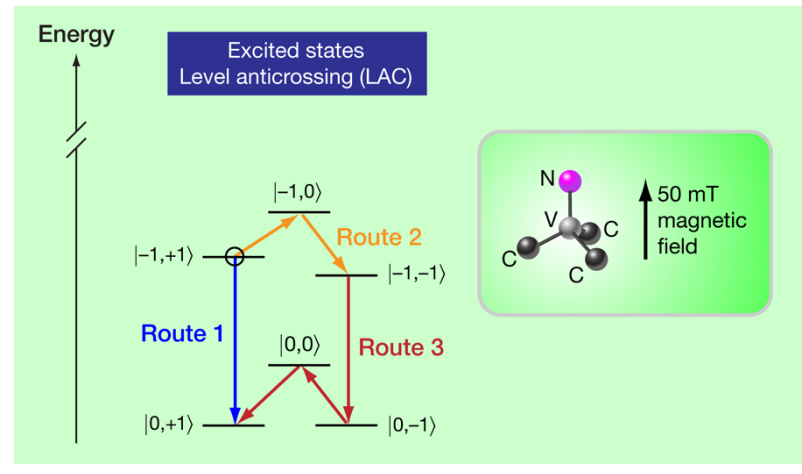

FIG. 1: Protocol for improving the readout signal from a single nitrogen-vacancy $\left(\mathrm{NV}^{-}\right)$center in diamond: $\mathrm{NV}^{-}$centers arise when a substitutional nitrogen atom $(\mathrm{N})$ is next to a vacancy $(\mathrm{V})$ as shown in the inset. The traditional readout technique used by most researchers to date is shown schematically as Route 1, which connects the two states that form the logical qubit without involving the nuclear spin state of the ${ }^{14} \mathrm{~N}$. However, by driving the system along route 2 and then 3 , the signal-to-noise is increased by $\sqrt{3}$. This technique is universal because it works for an $\mathrm{NV}^{-}$center in any local environment. The states are labeled by |electron spin state, ${ }^{14} \mathrm{~N}$ nuclear spin state $\rangle$. Routes 1 and 3 proceed via the excited states, and route 3 requires a level anticrossing (LAC) in the excited states, reached by the application of a $50 \mathrm{mT}$ magnetic field. (Illustration: Alan Stonebraker)

makes use of the ${ }^{14} \mathrm{~N}$ nuclear spin that is almost universally present in $\mathrm{NV}^{-}$centers [11. Matthias Steiner, Philipp Neumann, Johannes Beck, Fedor Jelezko, and Jörg Wrachtrup from Stuttgart University, Germany, have thereby demonstrated a signal-to-noise enhancement by a factor of $\sqrt{3}$. Most parade routes are designed to emphasize high visibility of the marchers and dignitaries rather than the shortest path from start to finish, 
and a similar approach has been applied to qubit readout by the Stuttgart group.

Two electron spin states are used as the logical qubit (or the magnetic sensor), and each of these is split into three by the $I=1^{14} \mathrm{~N}$ spin as shown in Fig. 11 Spin selection rules limit the allowed transitions between the six levels and the traditional readout of the qubit state proceeds along route 1 from $|-1,+1\rangle$ to $|0,+1\rangle$ via the excited states. To enhance the readout signal, the energy levels are modified by applying a magnetic field of $50 \mathrm{mT}$ along the symmetry axis of the $\mathrm{NV}^{-}$center (see inset of Fig. 1). This brings the system to a level anticrossing (LAC) in the excited state where the two electron spin states have the same energy [12. The excited state is accessed by the application of light for the fluorescence readout, and the LAC permits transitions between the logical qubit states. With the $50 \mathrm{mT}$ field on, the application of light polarizes not only the electron spin but also the nitrogen nuclear spin $[13$.

To prepare for the enhanced readout, the system is coherently driven along route 2 (see Fig. 1) by the application of $\mathrm{rf}$ radiation. Then, as a result of the LAC and spin-selection rules, the fluorescent readout leads the system along route 3 . Route 3 is three times longer than route 1 , and each step proceeds via the excited states. Three times more signal is detected as a result of this indirect series of events. Taking the indirect route postpones the destruction of the original spin state information. This "destruction" of the information is actually useful to $\mathrm{NV}^{-}$researchers, as the system is left in a polarized spin state, which is a convenient starting state for these experiments 6 .

To carry out the enhanced readout, it was necessary to coherently manipulate the ${ }^{14} \mathrm{~N}$ nuclear spin (route 2), and the success of this shows that this spin could be a useful qubit also. The first demonstration of coherent control over a single ${ }^{14} \mathrm{~N}$ spin has been carried out simultaneously by researchers at Bates College, US [14. Their work also uses the LAC but describes the possibility of reading out single nuclear qubits that could be ${ }^{15} \mathrm{~N},{ }^{14} \mathrm{~N}$, or ${ }^{13} \mathrm{C}$, all via the $\mathrm{NV}^{-}$electron spin.

A complementary approach to room-temperature spin state readout of a single $\mathrm{NV}^{-}$center is simply to collect more of the fluorescent light emitted. This can be achieved by putting a nanocrystal of diamond into a cavity, which efficiently couples photons to the detector [15]. Combining a cavity experiment with one of the enhanced measurement protocols described here could allow researchers to ask what spin state a $\mathrm{NV}^{-}$is in at room temperature.

Beyond this goal lies the broader challenge of diamond quantum computing, which will require progress in scalable techniques for controllably coupling spins. The three-spin experiments performed already [16 are impressive but do not appear to scale up to many qubits. It may be possible to overcome this by using control spins to turn couplings on and off [17], or even by entangling distant qubits with photon measurements 18 .

The prospect of a room-temperature quantum computer in the solid state is likely to attract even more scientists to the $\mathrm{NV}^{-}$parade and the indirect readout route described by Steiner et al. may be part of the finale.

\section{References}

[1] D. Loss, and D. P. DiVincenzo, Phys. Rev. A 57, 120 (1998).

[2] B. E. Kane, Nature 393, 133 (1998).

[3] J. R. Maze et al., Nature 455, 644 (2008).

[4] G. Balasubramanian et al., Nature 455, 648 (2008).

[5] G. Balasubramanian et al., Nature Mat. 8, 383 (2009).

[6] J. Harrison, M. J. Sellars, and N. B. Manson, J. Lumines. 107, 245 (2004).

[7] E. van Oort, N. B. Manson, and M. Glasbeek, J. Phys. C 21, 4385 (1988).

[8] A. Gruber et al., Science 276, 2012 (1997).

[9] F. Jelezko et al., Appl. Phys. Lett. 81, 2160 (2002).

[10] L. Jiang et al., Science 326, 267 (2009).

[11] M. Steiner, P. Neumann, J. Beck, F. Jelezko, and J. Wrachtrup, Phys. Rev. B 81, 035205 (2010).

[12] G. D. Fuchs et al., Phys. Rev. Lett. 101, 117601 (2008).

[13] V. Jacques et al., Phys. Rev. Lett. 102, 057403 (2009).

[14] B. Smeltzer, J. McIntyre, L. Childress, arXiv:0909.3896v1 (quant-ph) (2009).

[15] Y. S. Park, A. K. Cook, and H. L. Wang, Nano Lett. 6, 2075 (2006).

[16] P. Neumann et al., Science 320, 1326 (2008).

[17] A. M. Stoneham, A. H. Harker, and G. W. Morley, J. Phys. Cond. Matter 21, 364222 (2009).

[18] S. C. Benjamin et al., New J. Phys. 8, 9 (2006). 


\section{About the Author}

\section{Gavin W. Morley}

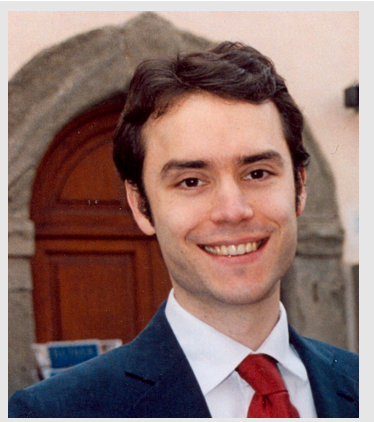

Gavin Morley studied physics at the University of Oxford and obtained his D.Phil. from the same university for "Designing a quantum computer based on pulsed electron spin resonance (ESR)." During post-doctoral work at the National High Magnetic Field Laboratory in Tallahassee, Florida, he developed a pulsed ESR spectrometer operating at higher magnetic fields and frequencies than any other currently operational worldwide. He was awarded an 1851 Research Fellowship to support his research at University College London within the London Centre for Nanotechnology and the Department of Physics and Astronomy. His recent projects focus on spins in silicon. 